\title{
Combinatorial Optimization with Noisy Inputs: How Can We Separate the Wheat from the Chaff?
}

\author{
Peter Widmayer \\ Institute of Theoretical Computer Science, ETH Zürich, Switzerland \\ widmayer@inf .ethz.ch
}

We postulate that real world data are almost always noisy, and an exact solution to a noisy input instance of a combinatorial optimization problem is not what we really want. Noise, or input data uncertainty, has a variety of reasons, such as for instance the need to estimate data based on imprecise measurements or on predictions (drawn from historical data and expected modifications). There is a variety of popular ways to deal with this uncertainty problem. In lucky cases in which the input data distribution is known, one might aim at obtaining a solution that is good in expectation. A different, promising way to handle uncertainty is based on the availability of a discrete set of possible problem instances (sometimes reflecting a distribution), so-called scenarios. A solution must be proposed for a set of scenarios as input, and thereafter a single scenario reveals itself as the actual one. The goal here is to achieve a high quality of the proposed solution with respect to the revealed scenario. Stochastic programming can be used to aim at a good solution in expectation that is feasible for most scenarios. In contrast, robust optimization most often aims at a solution that is feasible in all scenarios and has smallest worst case cost. In any case, uncertainty is considered a curse, a burden, a difficult problem that needs to be dealt with at extra computational cost.

We advocate to consider uncertainty a blessing that allows us to arrive at solutions that are more meaningful for the real world than if we just pretended that data were certain. We might even hope that taking uncertainty into account can sometimes allow for more efficient computations, because no attention needs to be paid to notoriously bad, artificial problem instances, and to the very last minor detail in the data. Towards this end, we advocate a way to ignore the noisy part in uncertain data, while preserving the meaningful part, that is, to separate the information in the data from the noise. We require simply two input instances for this purpose, and we show how to extract the information in these instances. Whenever an instance generator with a strongly peaked distribution hides behind both inputs, our approach shall return a close to optimum solution. We demonstrate the approach at a few simple algorithmic problems, such as finding a minimum in a set of numbers, a maximum subarray, a shortest path in a graph, and a minimum spanning tree. We discuss implications of the approach on the algorithmic complexity of these problems.

This talk reports about joint work with Joachim Buhmann, Matus Mihalak, and Rasto Sramek. 